\title{
Spatial distribution of mechanical properties in Pseudomonas aeruginosa biofilms, and their potential impacts on biofilm deformation
}

\author{
Juan Pablo Pavissich ${ }^{1}$, Mengfei $\mathrm{Li}^{2}$, and Robert Nerenberg ${ }^{2}$ \\ ${ }^{1}$ Universidad Adolfo Ibanez \\ ${ }^{2}$ University of Notre Dame
}

October 6, 2020

\begin{abstract}
The mechanical properties of biofilms can be used to predict biofilm deformation, for example under fluid flow. We used magnetic tweezers to spatially map the compliance of Pseudomonas aeruginosa biofilms at the micron scale, then used modeling to assess its effects on biofilm deformation. Biofilms were grown in capillary flow cells with Reynolds numbers (Re) ranging 0.28 to 13.9, bulk dissolved oxygen (DO) concentrations from $1 \mathrm{mg} / \mathrm{L}$ to $8 \mathrm{mg} / \mathrm{L}$, and bulk calcium ion $\left(\mathrm{Ca}^{2+}\right)$ concentrations of 0 and $100 \mathrm{mg} \mathrm{CaCl} / 2$ L. Higher Re numbers resulted in more uniform biofilm morphologies. The biofilm was stiffer at the center of the flow cell than near the walls. Lower bulk DO led to more stratified biofilms. Higher $\mathrm{Ca}^{2+}$ led to increased stiffness and more uniform mechanical properties. Using the experimental mechanical properties, fluid-structure interaction models predicted up to $64 \%$ greater deformations for heterogeneous biofilms, compared to a homogeneous biofilms with the same average properties. However, the error depended on the biofilm morphology and flow regime. Our results show significant spatial mechanical variability exists at the micron scale, and that this variability can potentially affect biofilm deformation. The average mechanical properties, provided in many studies, should be used with caution when predicting biofilm deformation.
\end{abstract}

\section{Introduction}

Biofilms are aggregates of microbial cells embedded in a matrix of extracellular polymeric substances (EPS) (Hans-Curt Flemming \& Wingender, 2010; Hall-Stoodley et al., 2004). They are ubiquitous in clinical, environmental, and industrial systems and can cause human infections, foul water filtration membranes, and promote corrosion in pipes (Costerton et al., 1995; Hall-Stoodley et al., 2004). Biofilms also can play beneficial roles, for example in environmental treatment processes. Thus, biofilm removal may be sought in some cases, and its retention in others.

Biofilm formation, persistence, deformation, and detachment are largely determined by the biofilm mechanical properties (Kundukad et al., 2016; Powell et al., 2013; Boudarel et al., 2018; Gloag et al., 2019). For example, biofilms' viscoelastic nature help them dissipate stress from fluid flow, preventing detachment (Stoodley et al., 1999). The characterization of biofilm mechanical properties is key to predicting biofilm deformation and detachment (Klapper et al., 2002).

Biofilm mechanical properties can be influenced by a variety of factors, including nutrient concentrations and microbial growth rates (Paul et al., 2012; Van Loosdrecht et al., 2002), microbial composition (Abriat et al., 2020; Kim et al., 2020; Yannarell et al., 2019), biofilm age (Hwang et al., 2014; Shen et al., 2016), hydrodynamic conditions (Dunsmore et al., 2002; Thomen et al., 2017), multivalent cation concentration (Ahimou et al., 2007; Jones et al., 2011; Lieleg et al., 2011), temperature (Pavlovsky et al., 2015; 2013), and pH values (Chen \& Stewart, 2000; Ho et al., 2013). For example, the microbial growth rates and ecological 
stratification, which are determined by the substrate profiles within the biofilm, can have a strong influence on biofilm mechanical strength (Rochex et al., 2009). Higher bulk oxygen concentrations and higher shear stresses were found to increase the strength of biofilms (Stoodley et al., 2002; Ahimou et al., 2007a; PellicerNàcher \& Smets, 2014). Also, the bulk calcium ion $\left(\mathrm{Ca}^{2+}\right)$ concentration caused biofilms to become thicker and denser, and to significantly decrease biofilm detachment episodes (Goode \& Allen, 2011).

While biofilms are commonly thought to be mechanically homogeneous, this may be an artifact generated by the use of bulk-scale techniques for their mechanical characterization (Safari et al., 2015). When microscale techniques were used, mechanical properties have been found to vary significantly within the biofilm (Böl et al., 2012).

Biofilms usually have temporal and spatial variations of mechanical properties. It has been widely recognized that the structure of biofilms becomes more stable over time. For example, older biofilms were found to be less affected by bubble disruptions, whereas younger biofilms were easily removed by air bubbles (Jang et al., 2017). Laspidou and Rittmann (2004b) hypothesized that biofilm increases its density over time due to consolidation, i.e., the filling of voids within the biofilm. In a study of the viscoelasticity of Pseudomonas aeruginosa biofilms (Gloag et al., 2018), various temporal changes were observed in different phenotypes. Increased stiffness or cohesive strength was observed over biofilm depth in several studies (Ahimou et al., 2007; Derlon et al., 2008; Olivier Galy et al., 2012). Spatial distribution of biofilm stiffness was also found in P. aeruginosa biofilms (Hunt et al., 2004; Karampatzakis et al., 2017).

In order to understand the spatial distribution of biofilm mechanical properties, microscale techniques can be used. Microscale techniques include microindentation compression (Cense et al., 2006), dedicated microcantilever (Aggarwal et al., 2010; Poppele \& Hozalski, 2003), atomic force microscopy (AFM) indentation (Arce et al., 2009; Volle et al., 2008), and microbead force spectroscopy (Lau et al., 2009). However, all these methods are invasive and can compromise the biofilm integrity.

In recent years, novel microrheological techniques have been developed (Birjiniuk et al., 2014; Cao et al., 2016; Galy et al., 2012; Karampatzakis et al., 2017; Thomen et al., 2017). In particular, magnetic actuation with magnetic tweezers, coupled with magnetic microparticles, may be ideal (Galy et al., 2014; Galy et al., 2012; Zrelli et al., 2013). This technique can overcome the limitations of other microscale techniques by using strong forces and in-situ measurements. With the displacement of magnetic particles, which are added to the media during initial growth, biofilm properties can be mapped spatially. For example, Galy et al. (2012) used magnetic tweezers and found that stiffness measurements at different locations in a biofilm ranged over of two orders of magnitude, even locally. This indicates the importance of localized spatial measurements in the study of biofilm mechanical properties. Nevertheless, the mechanical heterogeneity of biofilms has received little attention.

Mathematical models describing biofilm mechanical behavior can improve our understanding of biofilm structures and properties (Böl et al., 2012). Such models include the simulation of biofilm deformation under applied stress (Li et al., 2020; Picioreanu et al., 2018; Picioreanu et al., 2001; Towler et al., 2007). However, the mechanical properties are typically assumed to be homogeneous and are based on large-scale measurements. It is not clear whether mechanical heterogeneity is significant, and whether it should be considered for accurate predictions of biofilm deformation.

As discussed above, very few studies have addressed the spatial distribution and variability of mechanical properties of biofilms in a non-destructive fashion (Cao et al., 2016; Galy et al., 2012; Karampatzakis et al., 2017). Microscale heterogeneities could have important impacts on biofilm formation and behavior (Böl et al., 2012), yet the impact of this variability has not been explored.

In this study, we used magnetic tweezers to determine the spatial distribution of biofilm mechanical properties for different flow conditions, dissolved oxygen and $\mathrm{Ca}^{2+}$ concentrations in $P$. aeruginosa biofilms. $P$. aeruginosa is commonly used as a model species for biofilm research, as it is an important human pathogen and also widespread in the environment. We then used a fluid-structure interaction mathematical model to evaluate the mechanical behavior of a biofilm (i.e., biofilm deformation) considering average mechanical 
properties versus the heterogeneity observed in the experiments.

\section{Methods}

\section{Biofilm growth conditions}

P. aeruginosa ATCC strain 15692 (PAO1), tagged with green fluorescent protein (GFP), was used for biofilm growth (Shrout et al., 2006). The bacterial strain was grown overnight to an optical density of 0.4-0.5 $\left(\mathrm{OD}_{600}\right)$ in Luria-Bertani $(\mathrm{LB})$ broth at $37^{\circ} \mathrm{C}$ on an orbital shaker. A $1 \mathrm{~mL}$ aliquot of the culture was mixed with red fluorescent magnetic beads (Dynabeads M-270 Amine, Invitrogen, Carlsbad, CA), with a $2.8 \mu \mathrm{m}$ diameter, at a final concentration of $2.5 \times 10^{6}$ particles $/ \mathrm{mL}$. These beads are superparamagnetic, hydrophilic, and contain surface amino groups that form covalent bonds with cell and EPS components. The hydrophilic surface ensures non-specific binding to the biofilm. The superparamagnetic behavior allows for high levels of magnetization and the absence of magnetization without an external magnetic field. As a control, fluorescent non-magnetic particles of $1 \mu \mathrm{m}$ diameter were used (Fluoresbrite 18660, Polysciences, Germany).

Biofilms were grown in borosilicate glass capillaries with a $1 \mathrm{~mm}$ internal side and $150 \mu \mathrm{m}$ wall thickness (Friedrich and Dimmock Inc., Millville, NJ). The capillaries were inoculated with the mixed suspension and kept under static conditions for $2 \mathrm{~h}$ before starting a continuous flow of $10 \% \mathrm{LB}$ for the growth period. Flow was provided using a syringe pump (Kd Scientific, KDS-220, Holliston, MA). Flow rates were kept between 0.1 and $5 \mathrm{~mL} / \mathrm{h}$ to maintain Reynolds numbers (Re) between 0.28 and 13.9 (laminar flow). Biofilms were grown with dissolved oxygen (DO) concentrations of either 8 or $1 \mathrm{mg} / \mathrm{L}$, and with or without a $\mathrm{Ca}^{2+}$ addition of 100 $\mathrm{mg} / \mathrm{L}$ as $\mathrm{CaCl}_{2}$. Anin-situ magnetic actuation technique was then applied to non-destructively estimate the mechanical behavior of the biofilm after 5 days of growth. The general procedures were followed according to Galy et al. (2012). The detailed information of the magnetic tweezers and force calibration procedure is provided in Supporting Information Material (SI).

\section{Microscopy and particle tracking}

A confocal laser scanning microscopy (CLSM) (Nikon 90i A1R, Melville, NY) equipped with a 40x Plan Fluor WD 0.2 objective was used to image the biofilm and particles large-spectrum fluorescence signals. Typically, the first examined plane was located between 5 to $10 \mu \mathrm{m}$ above the capillary bottom. A z-stack with a depth step size of $0.5 \mu \mathrm{m}$ was collected to verify biofilm thickness and abundance of magnetic beads. For a given plane, particle motion upon magnetic force application was recorded at a scanning speed of 30 frames per second (fps) over a period of $20 \mathrm{~s}$, and further processed using a particle tracking software (ImageJ, NIH, Bethesda, MD). About 3 to 4 fields of $250 \mu \mathrm{m} \times 250 \mu \mathrm{m}$ (with each field) were collected along the capillary width before changing the plane (z-direction) (Figure 1). After each field was obtained, the field view was changed in the x-direction. Vertical planes were analyzed every $10 \mu \mathrm{m}$ across the height of the biofilm. About 10-15 particles were analyzed per field. A more visual description of the procedure can also be found in Galy et al. (2014).

\section{Creep curves and mechanical behavior analysis}

\section{Hosted file}

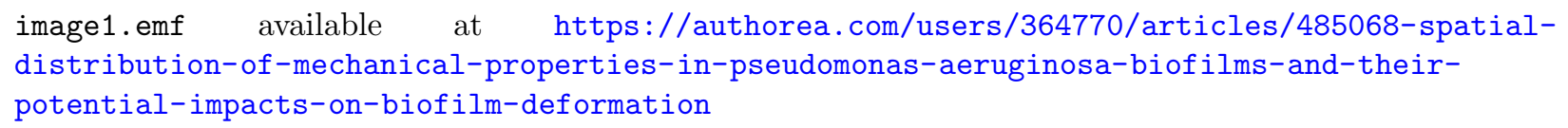

Using particle tracking, particle positions in each frame for the acquired images were obtained. The image acquisition frequency yielded particle trajectories or displacement as a function of time. Individual particle creep curves, giving material strain versus time, were plotted by converting the displacement curves into creep compliance curves (total compliance as a function of time $J(t)$ ), knowing the probe deflection $d(t)$ and the applied force $F$, according to the compliance formula (Schnurr, Gittes, MacKintosh, \& Schmidt, 1997): , which gives the relation between material strain and applied stress for a probe of radius $R$ (the microbead) 
embedded in an incompressible viscoelastic medium. The compliance $(J(t))$ of the material is the inverse of elastic modulus and equal to the ratio of strain to stress, i.e., the deformation for a given stress. Thus, a higher compliance indicates a more flexible material. The absolute force from the magnetic field acting on the magnetic particles was determined by a purely viscous mixture of $99.5 \%$ glycerol with a concentration of $2 \times 10^{8}$ particles $/ \mathrm{mL}$ (see SI).

\section{Modeling biofilm with heterogeneous mechanical properties}

To study the impacts of mechanical heterogeneity on biofilm deformation, a two-dimensional (2D) continuum biofilm model was implemented using COMSOL Multiphysics (COMSOL v4.4, Comsol Inc, Burlington, MA) with finite element method. A fluid-structure interaction (FSI) model using the arbitrary LagrangianEulerian (ALE) approach was applied to simulate the deformation of biofilms under fluid flow. The biofilm was considered either a purely linear elastic material with elastic compliance varying with depth in the biofilm, as per the experimental data, or a homogenous material, using the average of the experimental data. The system included a solid biofilm subdomain, and a bulk liquid subdomain. Two arbitrary biofilm structures were considered: a biofilm colony and a mushroom-like tower. The spatial distribution of mechanical properties was set in the biofilm structure using the experimental data obtained for the growth under 0.1 $\mathrm{mL} / \mathrm{h}$ flow rate, DO saturation, and no $\mathrm{Ca}^{2+}$ addition, with an elastic modulus (E) range of 0.005-0.05 $\mathrm{Pa}$, as calculated from the compliance $(J)$ determinations. The averaged elastic modulus of the complete heterogeneous biofilm structure was computed over the biofilm domain and then implemented into the homogeneous model. Time-dependent simulation was performed for 2-3 seconds until obtaining a steady-state solution. Biofilm deformations at $1.5 \mathrm{~s}$ were reported. A detailed description of the FSI model is provided in the SI.

\section{Results and Discussion}

We used magnetic microbead actuation together with confocal fluorescence microscopy to assess the spatial distribution of mechanical properties in GFP-tagged P. aeruginosa PAO1 biofilms. Creep curves showed the type of mechanical response ranging from elastic to viscoelastic. Non-magnetic beads did not show significant motion in the respective control at distances greater than $5 \mu \mathrm{m}$. In the local viscoelastic environments, the deformation observed for the loading time was dominated by elastic compliance, i.e., the viscous part of the deformation was lower that the elastic initial deformation. Also, about 20 to $30 \%$ of the particles (depending on the condition) showed purely elastic response. Thus, the analysis was focused on the spatial distribution of the elastic compliance $J$ for each biofilm growth condition. From $J$, the elastic modulus (E) can be obtained as its reciprocal and applied for mathematical simulations.

\section{Effect of shear stress}

The effect of flow-induced shear stress on the mechanical properties was studied by growing biofilms under different flow rates. The distribution of compliance values in different directions is shown in Figure 2, for biofilms grown under $0.1,1$ and $5 \mathrm{~mL} / \mathrm{h}(\mathrm{Re}=0.28,2.8$ and 13.9), respectively. Biofilms grown at the higher flow rates of 1 and $5 \mathrm{~mL} / \mathrm{h}$ were thinner (approximately 80-150 $\mu \mathrm{m}$ ) than at $0.1 \mathrm{~mL} / \mathrm{h}$ (approximately 150$250 \mu \mathrm{m}$ ). The spatial distribution of compliance revealed significant variability in mechanical properties. Higher heterogeneity was observed for the lower flow rates, with compliance values differing by as much as two orders of magnitude within the same biofilm (Figure 2). The distribution of compliance showed a significant correlation with biofilm depth $(\mathrm{Z})$, some dependence across the capillary width $(\mathrm{X})$, and was variable but not stratified across the capillary length $(\mathrm{Y})$.

The lowest flow rate, $0.1 \mathrm{~mL} / \mathrm{h}(\mathrm{Re}=0.28)$, produced the greatest vertical heterogeneity of compliance, with more elastic areas at the biofilm exterior and more rigid areas at the base. For the $1 \mathrm{~mL} / \mathrm{h}$ flow rate $(\mathrm{Re}=2.8)$, a more evenly layered structure was observed. The biofilm grown at $5 \mathrm{~mL} / \mathrm{h}(\mathrm{Re}=13.9)$ showed high rigidity throughout, with a mean compliance value as low as $0.02 \mathrm{~m}^{2} / \mathrm{N}(\mathrm{E}=50 \mathrm{~Pa})$. Increasing the flow shear stress decreased the average vertical compliance from values of $2 \mathrm{~m}^{2} / \mathrm{N}$ to $0.02 \mathrm{~m}^{2} / \mathrm{N}$ (elastic moduli range of 
0.5-50 Pa), producing more rigid structures. Similar results were observed in E. colibiofilms in a previous study using the same experimental technique (Galy et al., 2012). These results were observed for low shear stresses during growth (Re from $0.28-13.9$ corresponds to a shear stress of $0.22-11 \mathrm{mPa}$ ). Thus, the shear stress during growth was not expected to cause deformation, i.e., the elastic modulus is high compared to the wall shear stress in the channel. This suggests that the observed biofilm stiffening may be explained by a biological adaptation to low shear rather than a physical remodeling due to shear stress (Galy et al., 2012).

The compliances of biofilm basal layers $(0-20 \mu \mathrm{m})$ were almost the same for the different flow rates, despite greater differences in the outer layers. This suggests the mechanical properties near the biofilm base were not significantly affected by shear. This may be because the outer layer of the biofilm is more affected by the bulk conditions (Even et al., 2017). It also may be due to the lower biological activity at the base, leading to lower net growth rates compared to the upper layer. The deeper layers of biofilms are known to consolidate and became more dense over time (Laspidou \& Rittmann, 2004a).

The compliance across the capillary width (x-direction) showed a lower value near the center of the capillary channel. Result showing increasing stiffness in the center of biofilms have been reported by others (Karampatzakis et al., 2017). The variation of compliance along the capillary width may be caused by the shear force distribution. Stiffer biofilms in the middle of the flow channel correlate with the higher shear stress condition, while lower shear near the edges led to a softer biofilm. This is consistent with the findings from Thomen et al. (2017) who found that the development of biofilm had a strong relationship with the spatial distribution of shear stress.

The non-uniformity of biofilm mechanical properties can be correlated with the differences in concentrations of biofilm constituents, due to changes in EPS density or water content (Wilking et al., 2011). For instance, different EPS polysaccharides, such as Psl and Pel (Yang et al., 2011), produced by biofilm microorganisms such as $P$. aeruginosa can either increase the elasticity or increase the viscosity of biofilms, respectively (Chew et al., 2014; Friedman \& Kolter, 2004). Thomen et al. (2017) suggested that surrounding flows may partially wash out extracellular molecules secreted by cells. Based on the elastic results in our study, it is possible that shear flow removed bacteria cells instead of extracellular molecules, leading a higher stiffness under high shear stress. Although relatively low shear was used in the experiments, it is likely that continuous and higher shear stresses would result in an increasing production of EPS components such as Psl, increasing the strength of the matrix. Previous work also found an influence of hydrodynamics on the levels of quorum sensing molecules (Timp et al., 2009). Quorum sensing impacts gene expression and often promotes and regulation biofilm formation and EPS production. Therefore, it is also possible that shear stress changed the biofilm elasticity due to its impacts on quorum sensing.

\section{Effect of dissolved oxygen}

The effects DO on biofilm mechanical properties were studied for two flow rates, $0.1 \mathrm{~mL} / \mathrm{h}(\mathrm{Re}=0.28)$ and 5 $\mathrm{mL} / \mathrm{h}(\mathrm{Re}=13.9)$, using the methodology previously described. Compared to the biofilms obtained under DO saturation $(8 \mathrm{mg} / \mathrm{L})$, biofilms grown at a DO of $1 \mathrm{mg} / \mathrm{L}$ showed similar thickness but were more compact (less rough) (Figure 3). The vertical distribution of compliance values for these biofilms after 5 days of growth is shown in Figure 3.

At equal flow rates, biofilms displayed higher compliance values at lower DO levels. Similar to biofilms at high DO, the biofilms at low DO produced a wider range of compliance values flow for the lowest flow rates $(\mathrm{Re}=0.28)$. The outer biofilm layers had a higher compliance than the basal layers (Figure 3$)$. Interestingly, the highest flow rate $(\mathrm{Re}=13.9)$ also produced a layered biofilm, not as stiff and uniform as the one with high DO. The average compliance at $\mathrm{Re}=13.9$, around $0.6 \mathrm{~m}^{2} / \mathrm{N}$, was lower than for $\mathrm{Re}=0.28$, around 1.2 $\mathrm{m}^{2} / \mathrm{N}$. These results show the same trend discussed above, that increased shear stress leads to increased biofilm stiffness.

Higher compliances were observed in the superficial biofilm under low DO conditions. Previous research revealed a correlation between DO and growth rate and EPS production (Applegate \& Bryers, 1991; Laspidou 
\& Rittmann, 2004b). Laspidou and Rittmann (2004) used modeling to infer that consolidation in the bottom layers resulted in higher density and lower porosity. Lower production of polysaccharides was also associated with oxygen-limited conditions (Ahimou et al., 2007; Applegate \& Bryers, 1991). Under different bulk DO conditions, oxygen depletion with depth caused a variation of cell density and EPS production, which increased the heterogeneity of mechanical properties. As mentioned above, higher compliance in the superficial biofilm at low DO may be caused by low growth rate of cells. Furthermore, several studies have demonstrated that the oxygen gradients in biofilms are determined by the biological activity (Bridier et al., 2015; Xu et al., 1998). Thus, biofilm properties in the bottom layer are not strongly correlated with the oxygen concentrations in the bulk. The consistency of compliance values in the bottom layer was likely due to the lack of growing cells and anoxic conditions, regardless of the DO in the bulk liquid.

\section{Effect of divalent cations}

The compliance profile for biofilms grown at the lowest growth flow rate $(\mathrm{Re}=0.28)$ was determined for presence of $\mathrm{Ca}^{2+}$, as shown in Figure 4. In both cases the compliance values decreased, especially in the DO-saturated condition, where instead of the widely distributed compliance observed without $\mathrm{Ca}^{2+}$ addition, the results showed a reduced spread in values and a more homogeneous compliance profile. The change in heterogeneity can be correlated with the chemical effects of $\mathrm{Ca}^{2+}$, which form a bridge between negatively charged moieties in the EPS (Flemming \& Wingender, 2001). $\mathrm{Ca}^{2+}$ has been shown to promote ordered protein helices, and has a strong affinity for metal ions (Sehar et al., 2016; Sutherland, 2001). The change of compliance was due to the $\mathrm{Ca}^{2+}$, which promoted a stronger and stiffer biofilm matrix. Previous studies showed that $\mathrm{Ca}^{2+}$ could stimulate the development of thick and compact biofilms, with increased mechanical stability and elastic modulus (Ahimou et al., 2007; Körstgens et al., 2001; Shen et al., 2018). The results suggest that the development of stiffer structures was likely to be a consequence of high degrees of crosslinking.

The presence of $\mathrm{Ca}^{2+}$ led to much thicker biofilms when the bulk DO was high, and slightly thicker biofilms when the bulk DO was low. Sehar et al. (2016) concluded that the addition of $\mathrm{Ca}^{2+}$ could increase the cell density and thicken the biofilm. Thus, the formation of mechanically more stable biofilms in the presence of $\mathrm{Ca}^{2+}$ may be due to the strong cationic bridging between bacterial cells and EPS polymers. This is consistent with results obtained with other multivalent cations that play the same role as $\mathrm{Ca}^{2+}$, such as $\mathrm{Cu}^{2+}, \mathrm{Mg}^{2+}$, and $\mathrm{Fe}^{3+}$ (Beech \& Sunner, 2004).

\section{Modeling the impacts of mechanical property heterogeneity}

Past research has mainly assessed the mechanical properties of biofilms with macroscale tools (e.g., shear rheometry), characterizing an entire biofilm with one reading. Other studies have characterized only the outer layers of the biofilm (e.g., microindentation). Also, few modeling studies have considered mechanical heterogeneity. To illustrate the potential impacts of mechanical heterogeneity on biofilm behavior (i.e., biofilm deformation), as well as the potential differences in assuming biofilms have homogeneous elastic properties, we used a 2D FSI model to simulate the mechanical responses of two arbitrary biofilm morphologies. We arbitrarily assigned the experimental observed z-variations of mechanical properties from this study. Two biofilm morphologies were considered: a biofilm cluster or colony, and mushroom-like biofilm tower (Figure $5 \mathrm{a}$ and $5 \mathrm{~b})$.

The biofilm structures were modeled as purely linear-elastic solids, i.e., without viscous behavior. The initial morphologies were arbitrary, and it was assumed that the timescales were short enough that morphology changes due to growth or decay could be neglected. Thus, the model captures the short-term behavior when exposed to fluid flow. For each case, the deformations were compared for uniform mechanical properties and properties varying in the $\mathrm{z}$ direction per one of our experimental results.

Figure 5a and 5b shows 2D plots of the fluid velocities, von Mises stresses in the biofilm, and initial and final biofilm positions. The velocity of biofilm colony simulation $(\mathrm{Re}=250)$ was two orders of magnitude higher 
than that of biofilm tower simulation $(\mathrm{Re}=1)$ since the tower shape is more easily deformed. The original and deformed biofilm structures are shown as white outlines and colored surfaces in Figure 5a and 5b.

To evaluate the impact of mechanical heterogeneity on biofilm deformation, the displacement of the biofilm colony was plotted for as a function of biofilm depth (z direction) (Figure 5a and c). For the biofilm colony (Figure 5c), the heterogeneous biofilm suffered larger deformation. The more deformable top layer in the heterogeneous biofilm showed three times greater deformation ( $55 \mu \mathrm{m}$ at the top) than the homogeneous biofilm colony (20 $\mathrm{m}$ at the top). This is a $64 \%$ increase in deformation. For the mushroom-like biofilm tower (Figures $5 \mathrm{~b}$ and d), results showed larger deformations (70 $\mu \mathrm{m}$ at top) for the homogeneous biofilm than for the heterogeneous biofilm ( $55 \mu \mathrm{m}$ at top), a difference of $22.8 \%$.

The modeling simulations show that, even when the spatial distribution of elastic parameters is the same, the effect of mechanical heterogeneity on overall deformations can vary depending on the biofilm morphology. The assumption of homogeneous mechanical properties can lead to significant differences in deformation predictions.

Possible mechanisms and implications of biofilm mechanical heterogeneity

Our experimental results showed that $P$. aeruginosa biofilms have a heterogeneous distribution of mechanical properties, with a wide range of values. Other researchers have explored the mechanical non-uniformity both experimentally (Birjiniuk et al., 2014; Cao et al., 2016; Galy et al., 2012; Karampatzakis et al., 2017) and theoretically (Bridier et al., 2015; Even et al., 2017; Laspidou et al., 2014). The spatial distribution of biofilm mechanical properties was also found to vary with environmental conditions. The overall impacts of chemical, biological, and physical factors from the environment and biofilm itself explain the mechanical heterogeneity of the biofilm.

Stewart and Franklin (2008) concluded that mechanisms of biofilm heterogeneity including, but were not limited to, microscale chemical gradients, adaptation to local environmental conditions, stochastic gene expression, and genotypic variation. Another possible mechanism is that the growth of biofilms tended to be in the direction of minimal mechanical resistance, which lead to consistent gradients of stiffness, oxygen concentrations, nutrient concentrations, and growth rates (Even et al., 2017).

Biofilm heterogeneity has important practical implications, especially for biofilm control. Past research on the mechanical properties of biofilms show a wide range of results, possibly due to the wide variety of testing techniques at different length scales. In addition, spatial heterogeneity of mechanical properties, and differences in biofilm morphologies and structures, play key roles on the deformation of biofilms under fluid flow (Even et al., 2017; Trejo et al., 2013). Our modeling results show that the spatial heterogeneity of biofilm mechanical properties could lead to significant differences of the deformation, even with the same averaged value. For this reason, previous studies (Picioreanu et al., 2018; Stoodley et al., 1999) which backcalculated homogeneous mechanical properties using the experimental deformation should be viewed with caution.

Our modeling results show that the variability of mechanical properties can have different effects, depending on the biofilm morphology, spatial distribution of mechanical properties, and hydrodynamic profiles. Surface erosion and biofilm sloughing may occur more easily and frequently on more non-uniform biofilm colonies and more uniform mushroom-like biofilms.

In water and wastewater engineering, biofilms can play both beneficial and detrimental roles. For example, the accumulation of biofilm in biotreatment processes is critical to their good performance, while development of biofilm fouling layers in membrane filtration systems can greatly increase energy requirements. Thus, promoting accumulation of beneficial biofilms and promoting detachment of detrimental biofilms is an important practical need. Yet there are few tools available to predict biofilm behavior. This research provides both results on the variability of mechanical properties for a $P$. aeruginiosabiofilm, and also a tool to assess its impact on biofilm deformation.

In order to affect the mechanical stability of biofilms, the fluid flow rates and shear, DO concentrations, and 
concentration of divalent cations are important variables. Our results also suggest that biofilm initially may be more flexible and easy to detach at the base, but then become stiffer at the base as the biofilm matures. Thus, lighter and more frequent biofilm removal treatments may be more effective than ones that are less frequent and more intense. For longer term assessments of biofilm behavior, models should include growth and decay processes, and also the viscoelastic behavior of biofilms.

\section{Conclusions}

Magnetic tweezers were used to characterize the spatial distribution of elastic properties of $P$. aeruginosa biofilms for a range of conditions. Spatial heterogeneity was observed in all three-dimensions, and environmental conditions had a significant impact on the spatial distribution. Higher shear resulted in a stiffer and more uniform distribution, possibly due to mechanical adaptation. Also, stiffer biofilms in the center of the flow cell were correlated with greater hydrodynamic shear. Lower bulk DO led to a more heterogeneous biofilm, probably due to the greater variation of biological activity with depth in the biofilm. The addition of $\mathrm{Ca}^{2+}$ in bulk liquid increased the average stiffness and resulted in more uniform biofilms. Further research should address the viscoelastic behavior, and the combination of elastic deformation, viscous deformation, and growth, as all of these may significantly impact biofilm deformation and morphology in the long term.

Using mathematical model for two different hypothetical biofilm morphologies under fluid flow, it was shown that the spatial heterogeneity of mechanical properties can lead to significant differences in biofilm deformation. This demonstrates that biofilm mechanical heterogeneity should be considered when predicting biofilm deformation. Conversely, it also should be taken into consideration when using biofilm deformation to infer its mechanical properties.

\section{Acknowledgements}

This research was funded by NSF Project 1605177. Partial support to JPP was provided by FONDECYT 11181107 and NID PIA/BASAL FB0002, and to ML by the CEST/Bayer Predoctoral Research Fellowship at the University of Notre Dame. The authors would like to thank the Notre Dame Center for Research Computing for the computational resources. We thank Joshua Shrout for providing the P. aeruginosa strain.

\section{References:}

Abriat, C., Enriquez, K., Virgilio, N., Cegelski, L., Fuller, G. G., Daigle, F., \& Heuzey, M.-C. (2020). Mechanical and microstructural insights of Vibrio cholerae and Escherichia coli dual-species biofilm at the air-liquid interface. Colloids and Surfaces B: Biointerfaces ,188, 110786.

Aggarwal, S., Poppele, E. H., \& Hozalski, R. M. (2010). Development and testing of a novel microcantilever technique for measuring the cohesive strength of intact biofilms. Biotechnology and Bioengineering ,105 (5), 924-934.

Ahimou, F., Semmens, M. J., Haugstad, G., \& Novak, P. J. (2007). Effect of protein, polysaccharide, and oxygen concentration profiles on biofilm cohesiveness. Applied and Environmental Microbiology ,73 (9), 2905-2910. https://doi.org/10.1128/AEM.02420-06

Ahimou, F., Semmens, M. J., Novak, P. J., \& Haugstad, G. (2007). Biofilm cohesiveness measurement using a novel atomic force microscopy methodology. Applied and Environmental Microbiology ,73 (9), 2897-2904.

Applegate, D. H., \& Bryers, J. D. (1991). Effects of carbon and oxygen limitations and calcium concentrations on biofilm removal processes.Biotechnology and Bioengineering , 37 (1), 17-25.

Arce, F. T., Carlson, R., Monds, J., Veeh, R., Hu, F. Z., Stewart, P. S., .. . Avci, R. (2009). Nanoscale structural and mechanical properties of nontypeable Haemophilus influenzae biofilms. Journal of Bacteriology , 191 (8), 2512-2520.

Beech, I. B., \& Sunner, J. (2004). Biocorrosion: towards understanding interactions between biofilms and metals. Current Opinion in Biotechnology , 15 (3), 181-186. 
Birjiniuk, A., Billings, N., Nance, E., Hanes, J., Ribbeck, K., \& Doyle, P. S. (2014). Single particle tracking reveals spatial and dynamic organization of the Escherichia coli biofilm matrix. New Journal of Physics , 16 . https://doi.org/10.1088/1367-2630/16/8/085014

Böl, M., Ehret, A. E., Bolea Albero, A., Hellriegel, J., \& Krull, R. (2012). Recent advances in mechanical characterisation of biofilm and their significance for material modelling. Critical Reviews in Biotechnology , 8551 (August 2015), 1-27. https://doi.org/10.3109/07388551.2012.679250

Boudarel, H., Mathias, J.-D., Blaysat, B., \& Grédiac, M. (2018). Towards standardized mechanical characterization of microbial biofilms: analysis and critical review. NPJ Biofilms and Microbiomes ,4 (1), 1-15.

Bridier, A., Sanchez-Vizuete, P., Guilbaud, M., Piard, J. C., Naïtali, M., \& Briandet, R. (2015). Biofilm-associated persistence of food-borne pathogens. Food Microbiology , 45 (PB), 167-178. https://doi.org/10.1016/j.fm.2014.04.015

Cao, H., Habimana, O., Safari, A., Heffernan, R., Dai, Y., \& Casey, E. (2016). Revealing region-specific biofilm viscoelastic properties by means of a micro-rheological approach. Npj Biofilms and Microbiomes, 2 (1), 5. https://doi.org/10.1038/s41522-016-0005-y

Cense, A. W., Peeters, E. A. G., Gottenbos, B., Baaijens, F. P. T., Nuijs, A. M., \& van Dongen, M. E. H. (2006). Mechanical properties and failure of Streptococcus mutans biofilms, studied using a microindentation device. Journal of Microbiological Methods ,67 (3), 463-472. https://doi.org/10.1016/j.mimet.2006.04.023

Chen, X., \& Stewart, P. S. (2000). Biofilm removal caused by chemical treatments. Water Research , 34 (17), 4229-4233. https://doi.org/10.1016/S0043-1354(00)00187-1

Chew, S. C. hue., Kundukad, B., Seviour, T., van der Maarel, J. R. C., Yang, L., Rice, S. A., .. Kjelleberg, S. (2014). Dynamic remodeling of microbial biofilms by functionally distinct exopolysaccharides. MBio , 5 (4), e01536-e01514. https://doi.org/10.1128/mBio.01536-14

Costerton, J. W., Lewandowski, Z., Caldwell, D. E., Korber, D. R., \& Lappin-Scott, H. M. (1995). Microbial biofilms. Annual Reviews in Microbiology , 49 (1), 711-745.

Derlon, N., Massé, A., Escudié, R., Bernet, N., \& Paul, E. (2008). Stratification in the cohesion of biofilms grown under various environmental conditions. Water Research , 42 (8-9), 2102-2110. https://doi.org/10.1016/j.watres.2007.11.016

Dunsmore, B. C., Jacobsen, A., Hall-Stoodley, L., Bass, C. J., Lappin-Scott, H. M., \& Stoodley, P. (2002). The influence of fluid shear on the structure and material properties of sulphate-reducing bacterial biofilms. Journal of Industrial Microbiology and Biotechnology , 29 (6), 347-353.

Even, C., Marlière, C., Ghigo, J. M., Allain, J. M., Marcellan, A., \& Raspaud, E. (2017). Recent advances in studying single bacteria and biofilm mechanics. Advances in Colloid and Interface Science,247 (July), 573-588. https://doi.org/10.1016/j.cis.2017.07.026

Flemming, H-C, \& Wingender, J. (2001). Relevance of microbial extracellular polymeric substances (EPSs)Part I: Structural and ecological aspects. Water Science and Technology , 43 (6), 1-8.

Flemming, Hans-Curt, \& Wingender, J. (2010). The biofilm matrix.Nature Reviews. Microbiology , 8 (9), 623-633. https://doi.org/10.1038/nrmicro2415

Friedman, L., \& Kolter, R. (2004). Two genetic loci produce distinct carbohydrate-rich structural components of the Pseudomonas aeruginosa biofilm matrix. Journal of Bacteriology , 186 (14), 4457-4465.

Galy, O, Zrelli, K., Latour-Lambert, P., Kirwan, L., \& Henry, N. (2014). Remote Magnetic Actuation of Micrometric Probes for in situ 3D Mapping of Bacterial Biofilm Physical Properties. Journal of Visualized Experiments : JoVE , 87 (May), 1-12. https://doi.org/10.3791/50857 
Galy, Olivier, Latour-Lambert, P., Zrelli, K., Ghigo, J. M., Beloin, C., \& Henry, N. (2012). Mapping of bacterial biofilm local mechanics by magnetic microparticle actuation. Biophysical Journal ,103 (6), 14001408. https://doi.org/10.1016/j.bpj.2012.07.001

Gloag, E. S., Fabbri, S., Wozniak, D. J., \& Stoodley, P. (2019). Biofilm mechanics: implications in infection and survival.Biofilm, 100017.

Gloag, E. S., German, G. K., Stoodley, P., \& Wozniak, D. J. (2018). Viscoelastic properties of Pseudomonas aeruginosa variant biofilms.Scientific Reports , 8 (1), 9691.

Goode, C., \& Allen, D. G. (2011). Effect of calcium on moving-bed biofilm reactor biofilms. Water Environment Research ,83 (3), 220-232.

Hall-Stoodley, L., Costerton, J. W., \& Stoodley, P. (2004). Bacterial biofilms: from the natural environment to infectious diseases. Nature Reviews Microbiology, 2 , 95-108. https://doi.org/10.1038/nrmicro821

Ho, Y.-R., Li, C.-M., Yu, C.-H., Lin, Y.-J., Wu, C.-M., Harn, I.-C., . . Lu, C.-Y. (2013). The enhancement of biofilm formation in Group B streptococcal isolates at vaginal $\mathrm{pH}$. Medical Microbiology and Immunology , $202(2), 105-115$.

Hunt, S. M., Werner, E. M., Huang, B., Hamilton, M. A., \& Stewart, P. S. (2004). Hypothesis for the role of nutrient starvation in biofilm detachment. Applied and Environmental Microbiology ,70 (12), 7418-7425.

Hwang, G., Klein, M. I., \& Koo, H. (2014). Analysis of the mechanical stability and surface detachment of mature Streptococcus mutans biofilms by applying a range of external shear forces. Biofouling ,30 (9), 1079-1091.

Jang, H., Rusconi, R., \& Stocker, R. (2017). Biofilm disruption by an air bubble reveals heterogeneous age-dependent detachment patterns dictated by initial extracellular matrix distribution. Npj Biofilms and Microbiomes , 3 (1), 1-7.

Jones, W. L., Sutton, M. P., McKittrick, L., \& Stewart, P. S. (2011). Chemical and antimicrobial treatments change the viscoelastic properties of bacterial biofilms. Biofouling , 27 (2), 207-215. https://doi.org/10.1080/08927014.2011.554977

Karampatzakis, A., Song, C. Z., Allsopp, L. P., Filloux, A., Rice, S. A., Cohen, Y., ... Torok, P. (2017). Probing the internal micromechanical properties of Pseudomonas aeruginosa biofilms by Brillouin imaging. Npj Biofilms and Microbiomes , 3 (1), 20. https://doi.org/10.1038/s41522-017-0028-z

Kim, B., Perez-Calleja, P., Li, M., \& Nerenberg, R. (2020). Effect of predation on the mechanical properties and detachment of MABR biofilms. Water Research , 116289.

Klapper, I., Rupp, C. J., Cargo, R., Purvedorj, B., \& Stoodley, P. (2002). Viscoelastic fluid description of bacterial biofilm material properties. Biotechnology and Bioengineering , 80 (3), 289-296. https://doi.org/10.1002/bit.10376

Korstgens, V., Flemming, H. C., Wingender, J., \& Borchard, W. (2001). Influence of calcium ions on the mechanical properties of a model biofilm of mucoid Pseudomonas aeruginosa. Water Science and Technology : A Journal of the International Association on Water Pollution Research , 43 (6), 49-57. https://doi.org/10.1371/journal.pone.0091935

Kundukad, B., Seviour, T., Liang, Y., Rice, S. A., Kjelleberg, S., \& Doyle, P. S. (2016). Mechanical properties of the superficial biofilm layer determine the architecture of biofilms. Soft Matter ,12 , 57185726. https://doi.org/10.1039/C6SM00687F

Laspidou, C. S., Spyrou, L. A., Aravas, N., \& Rittmann, B. E. (2014). Material modeling of biofilm mechanical properties. Mathematical Biosciences , 251 (1), 11-15. https://doi.org/10.1016/j.mbs.2014.02.007 
Laspidou, Chrysi S, \& Rittmann, B. E. (2004a). Evaluating trends in biofilm density using the UMCCA model. Water Research ,38 (14-15), 3362-3372.

Laspidou, Chrysi S, \& Rittmann, B. E. (2004b). Modeling the development of biofilm density including active bacteria, inert biomass, and extracellular polymeric substances. Water Research ,38 (14), 3349-3361.

Lau, P. C. Y., Dutcher, J. R., Beveridge, T. J., \& Lam, J. S. (2009). Absolute Quantitation of Bacterial Biofilm Adhesion and Viscoelasticity by Microbead Force Spectroscopy. Biophysical Journal ,96 (7), 29352948. https://doi.org/10.1016/j.bpj.2008.12.3943

Li, M., Matouš, K., \& Nerenberg, R. (2020). Predicting biofilm deformation with a viscoelastic phase-field model: Modeling and experimental studies. Biotechnology and Bioengineering , 1-13. https://doi.org/10.1002/bit.27491

Lieleg, O., Caldara, M., Baumgärtel, R., \& Ribbeck, K. (2011). Mechanical robustness of Pseudomonas aeruginosa biofilms. Soft Matter , 7 (7), 3307-3314. https://doi.org/10.1039/c0sm01467b

Paul, E., Ochoa, J. C., Pechaud, Y., Liu, Y., \& Liné, A. (2012). Effect of shear stress and growth conditions on detachment and physical properties of biofilms. Water Research , 46 (17), 5499-5508.

Pavlovsky, L., Sturtevant, R. A., Younger, J. G., \& Solomon, M. J. (2015). Effects of temperature on the morphological, polymeric, and mechanical properties of Staphylococcus epidermidis bacterial biofilms.Langmuir , 31 (6), 2036-2042.

Pavlovsky, L., Younger, J. G., \& Solomon, M. J. (2013). In situ rheology of Staphylococcus epidermidis bacterial biofilms. Soft Matter , 9 (1), 122-131. https://doi.org/10.1039/C2SM27005F

Pellicer-Nàcher, C., \& Smets, B. F. (2014). Structure, composition, and strength of nitrifying membraneaerated biofilms. Water Research ,57, 151-161.

Picioreanu, C., Blauert, F., Horn, H., \& Wagner, M. (2018). Determination of mechanical properties of biofilms by modelling the deformation measured using optical coherence tomography. Water Research, 145 , 588-598.

Picioreanu, C., Van Loosdrecht, M. C. M., \& Heijnen, J. J. (2001). Two-dimensional model of biofilm detachment caused by internal stress from liquid flow. Biotechnology 83 Bioengineering , 72 (2), 205-218.

Poppele, E. H., \& Hozalski, R. M. (2003). Micro-cantilever method for measuring the tensile strength of biofilms and microbial flocs. Journal of Microbiological Methods , 55 (3), 607-615.

Powell, L. C., Sowedan, A., Khan, S., Wright, C. J., Hawkins, K., Onsøyen, E., .. Thomas, D. W. (2013). The effect of alginate oligosaccharides on the mechanical properties of Gram-negative biofilms.Biofouling , 29 (4), 413-421.

Rochex, A., Massé, A., Escudié, R., Godon, J. J., \& Bernet, N. (2009). Influence of abrasion on biofilm detachment: Evidence for stratification of the biofilm. Journal of Industrial Microbiology and Biotechnology , 36 (3), 467-470. https://doi.org/10.1007/s10295-009-0543-x

Safari, A., Tukovic, Z., Walter, M., Casey, E., \& Ivankovic, A. (2015). Mechanical properties of a mature biofilm from a wastewater system: from microscale to macroscale level. Biofouling , 31 (8), 651-664. https://doi.org/10.1080/08927014.2015.1075981

Schnurr, B., Gittes, F., MacKintosh, F. C., \& Schmidt, C. F. (1997). Determining microscopic viscoelasticity in flexible and semiflexible polymer networks from thermal fluctuations. Macromolecules ,30 (25), 7781-7792.

Sehar, S., Naz, I., Das, T., \& Ahmed, S. (2016). Evidence of microscopic correlation between biofilm kinetics and divalent cations for enhanced wastewater treatment efficiency. RSC Adv. ,6 (18), 15112-15120. https://doi.org/10.1039/C5RA21076C 
Shen, Y., Huang, C., Monroy, G. L., Janjaroen, D., Derlon, N., Lin, J., ... Nguyen, T. H. (2016). Response of Simulated Drinking Water Biofilm Mechanical and Structural Properties to Long-Term Disinfectant Exposure. Environmental Science and Technology , 50 (4), 1779-1787. https://doi.org/10.1021/acs.est.5b04653

Shen, Y., Huang, P. C., Huang, C., Sun, P., Monroy, G. L., Wu, W., .. Liu, W.-T. (2018). Effect of divalent ions and a polyphosphate on composition, structure, and stiffness of simulated drinking water biofilms. NPJ Biofilms and Microbiomes, 4 (1), 1-9.

Shrout, J. D., Chopp, D. L., Just, C. L., Hentzer, M., Givskov, M., \& Parsek, M. R. (2006). The impact of quorum sensing and swarming motility on Pseudomonas aeruginosa biofilm formation is nutritionally conditional. Molecular Microbiology , 62 (5), 1264-1277.

Stewart, P. S., \& Franklin, M. J. (2008). Physiological heterogeneity in biofilms. Nature Reviews Microbiology , 6 (3), 199-210. https://doi.org/10.1038/nrmicro1838

Stoodley, P., Cargo, R., Rupp, C. J., Wilson, S., \& Klapper, I. (2002). Biofilm material properties as related to shear-induced deformation and detachment phenomena. Journal of Industrial Microbiology and Biotechnology , 29 (6), 361-367.

Stoodley, P., Lewandowski, Z., Boyle, J. D., \& Lappin-Scott, H. M. (1999). The formation of migratory ripples in a mixed species bacterial biofilm growing in turbulent flow. Environmental Microbiology ,1 (5), 447-455. https://doi.org/emi55 [pii]

Stoodley, P., Lewandowski, Z., Boyle, J. D., \& Lappin-Scott, H. M. (1999). Structural deformation of bacterial biofilms caused by short-term fluctuations in fluid shear: An in situ investigation of biofilm rheology. Biotechnology and Bioengineering , 65 (1), 83-92.

Sutherland, I. W. (2001). Biofilm exopolysaccharides: A strong and sticky framework. Microbiology , 147 (1), 3-9. https://doi.org/10.1051/lait:2001108

Thomen, P., Robert, J., Monmeyran, A., Bitbol, A. F., Douarche, C., \& Henry, N. (2017). Bacterial biofilm under flow: First a physical struggle to stay, then a matter of breathing. PLoS ONE ,12 (4), 1-24. https://doi.org/10.1371/journal.pone.0175197

Timp, W., Mirsaidov, U., Matsudaira, P., \& Timp, G. (2009). Jamming prokaryotic cell-to-cell communications in a model biofilm. Lab Chip , 9 (7), 925-934. https://doi.org/10.1039/B810157D

Towler, B. W., Cunningham, A., Stoodley, P., \& McKittrick, L. (2007). A model of fluid-biofilm interaction using a Burger material law.Biotechnology and Bioengineering , 96 (2), 259-271.

Trejo, M., Douarche, C., Bailleux, V., Poulard, C., Mariot, S., Regeard, C., \& Raspaud, E. (2013). Elasticity and wrinkled morphology of Bacillus subtilis pellicles. Proceedings of the National Academy of Sciences , 110 (6), 2011-2016. https://doi.org/10.1073/pnas.1217178110

Van Loosdrecht, M. C. M., Heijnen, J. J., Eberl, H., Kreft, J., \& Picioreanu, C. (2002). Mathematical modelling of biofilm structures. Antonie van Leeuwenhoek , 81 (1-4), 245-256.

Volle, C. B., Ferguson, M. A., Aidala, K. E., Spain, E. M., \& Nunez, M. E. (2008). Spring constants and adhesive properties of native bacterial biofilm cells measured by atomic force microscopy. Colloids and Surfaces B: Biointerfaces , 67 (1), 32-40.

Wilking, J. N., Angelini, T. E., Seminara, A., Brenner, M. P., \& Weitz, D. A. (2011). Biofilms as complex fluids. MRS Bulletin ,36 (5), 385-391.

Xu, K. D., Stewart, P. S., Xia, F., Mcfeters, G. a, \& Huang, C. (1998). Spatial physiological heterogeneity in Pseudomonas aeruginosa biofilm is determined by oxygen availability. Applied and environmental microbiology , 64 (10), 4035-4039. 
Yang, L., Hu, Y., Liu, Y., Zhang, J., Ulstrup, J., \& Molin, S. (2011). Distinct roles of extracellular polymeric substances in Pseudomonas aeruginosa biofilm development. Environmental Microbiology ,13 (7), 1705-1717.

Yannarell, S. M., Grandchamp, G. M., Chen, S.-Y., Daniels, K. E., \& Shank, E. A. (2019). A dual-species biofilm with emergent mechanical and protective properties. Journal of Bacteriology, 201 (18), e00670-18.

Zrelli, K., Galy, O., Latour-Lambert, P., Kirwan, L., Ghigo, J. M., Beloin, C., \& Henry, N. (2013). Bacterial biofilm mechanical properties persist upon antibiotic treatment and survive cell death. New Journal of Physics , 15 . https://doi.org/10.1088/1367-2630/15/12/125026

\section{Hosted file}

Tweezers paper_Figure_BB.pdf available at https://authorea.com/users/364770/articles/485068spatial-distribution-of-mechanical-properties-in-pseudomonas-aeruginosa-biofilms-andtheir-potential-impacts-on-biofilm-deformation 